\title{
All-Garnet Magneto-Optical Photonic Crystals
}

\author{
A.M. Grishin and S.I. Khartsev \\ Department of Condensed Matter Physics, Royal Institute of Technology, SE-164 40 Stockholm-Kista, Sweden
}

We survey the properties of all-garnet magneto-optical (MO) heteroepitaxial film structures grown by pulsed laser deposition and rf-magnetron sputtering. 1D MO-photonic crystals (MOPCs) were composed of $\lambda / 4$ garnet layers alternating highly gyrotropic $\mathrm{Bi}_{3} \mathrm{Fe}_{5} \mathrm{O}_{12}(\mathrm{BIG})$ and $\mathrm{MO}$-passive rare earth gallium garnets. As designed, MOPCs' spectra exhibit optical stop band with the transmittance central peak caused by light localization in $\mathrm{N} / 2$ thick BIG cavity. The first all-garnet BIG/YIG MOPC showed 140\% enhancement of the Faraday rotation (FR) compared to a single layer BIG film [APL 84, 1438 (2004)]. Further improvement of MO-performance has been achieved due to the replacement of optically dense YIG by transparent Gd- (GGG), Sm- and novel La-Ga-garnets [APL 86, 141108 (2005); 87, 122504 (2005); 90, 191113 (2007); JAP 101, 053906 (2007)]. At the resonance wavelengths 750 (980) nm, specific FR $\theta_{\mathrm{F}}=-20.5(-7.3) \mathrm{deg} / \mu \mathrm{m}$ and MO-quality factor $Q=2\left|\theta_{\mathrm{F}}\right|$ /absorption $=66(43.6)$ deg represent the highest MOPC performance achieved so far. Respectively, this is 470 (810) \% and 31 (190) \% enhancement compared to a single layer BIG. MO-remanence (latching capability) has been engineered in the series of BIG:GGG(n:m) superlattices. Regular alternating of lattice mismatched garnet layers impedes the nucleation of misfit dislocations, preserves a long range coherent compressive strain through the whole multilayer thickness thus results in a strong uniaxial magnetic anisotropy. $2.5 \mathrm{\mu m}$ thick BIG:GGG(3:2) film at $678 \mathrm{~nm}$ shows $\mathrm{FR}= \pm 1.4 \mathrm{deg}$, transmittance $82 \%$, $92 \%$ squareness of magnetization loop, saturation and coercive fields as low as 56 and 25 Oe, respectively. Nanostructured garnets were used to build MO-visualizer and current driven MO-display [APL 88, 242504 (2006)].

Key words: bismuth iron garnet; pulsed laser deposition; rf-magnetron sputtering; heteroepitaxial multilayers; Faraday rotator; magneto-optical visualizer; magneto-optical display.

\section{Introduction}

Over the past decade materials with forbidden photonic band (photonic crystals) have formed a rapidly expanding niche of photonics. They have enormous potential for light guiding, filtering and switching, exceptional dispersion properties (superprism effect) as well as a potential to be integrated with CMOS devices on Si platform. Magnetic photonic band gap materials attract special interest since they possess nonreciprocal properties. ${ }^{1)}$

M. Inoue et al. fabricated and tested the first $1 \mathrm{D}$ MOPCs with $\mathrm{Ta}_{2} \mathrm{O}_{5} / \mathrm{SiO}_{2}$ dielectric mirrors and various MO-materials for the central optical cavity: polycrystalline $\mathrm{Bi}$-substituted dysprosium iron garnet (Bi:DyIG), Bi:YIG as well as granular $\mathrm{Co}^{-\mathrm{Sm}-\mathrm{O} \text { and }}$ Fe-Si-O magnetic layers (see ${ }^{2)}$ and references therein).

Significant breakthrough has been achieved using completely substituted bismuth iron garnet $\mathrm{Bi}_{3} \mathrm{Fe}_{5} \mathrm{O}_{12}$ as a Faraday rotator with a record Faraday rotation (FR) $\theta_{\mathrm{F}}=-8.4 \mathrm{deg} / \mu \mathrm{m} @ 633 \mathrm{~nm}$ and the peak value as high as - $27.9 \mathrm{deg} / \mathrm{\mu m} @ 537 \mathrm{~nm} .^{3)}$ Later, the first all-garnet $1 \mathrm{D}$ heteroepitaxial $\mathrm{Bi}_{3} \mathrm{Fe}_{5} \mathrm{O}_{12} / \mathrm{Y}_{3} \mathrm{Fe}_{5} \mathrm{O}_{12}$ PLD-grown MOPC 4) showed superior MO-properties: at the designed wavelength of $750 \mathrm{~nm}$ FR was increased by $140 \%$ while transmission decreased by just $16 \%$ compared with a single-layer BIG of equivalent thickness. In this paper we present results on fabrication and testing of all-garnet heteroepitaxial film structures containing $\mathrm{MO}$-active $\mathrm{Bi}_{3} \mathrm{Fe}_{5} \mathrm{O}_{12}$ (BIG) and transparent $\mathrm{Gd}_{3} \mathrm{Ga}_{5} \mathrm{O}_{12}$ (GGG), $\mathrm{Sm}_{3} \mathrm{Ga}_{5} \mathrm{O}_{12}$ (SGG), and $\mathrm{La}_{3} \mathrm{Ga}_{5} \mathrm{O}_{12}$ (LGG) layers.

\section{Heteroepitaxial film processing and crystalline structure}

The fabrication of epitaxial garnets was carried out according to the following outline. $\mathrm{KrF}$ excimer $248-\mathrm{nm}$ laser Compex-102 was used to ablate ceramic targets. Composite BIG target contained overstoichiometric ratio of $\mathrm{Bi}$ and $\mathrm{Fe}$ oxides $(\mathrm{Bi}: \mathrm{Fe}=3.15: 5)$, LGG target was made from the stoichiometric mixture of $\mathrm{La}_{2} \mathrm{O}_{3}$ and $\mathrm{Ga}_{2} \mathrm{O}_{3}$ oxides, while for GGG we used polycrystalline $\mathrm{Gd}_{3} \mathrm{Ga}_{5} \mathrm{O}_{12}$ ceramics. Both side polished GGG, $\mathrm{Ca}, \mathrm{Mg}, \mathrm{Zr}$ :GGG and $\mathrm{Gd}_{3} \mathrm{Sc}_{2} \mathrm{Ga}_{3} \mathrm{O}_{12}$ (111) and (001) single crystals were used as substrates. Synthesis of multilayered garnets was always performed at stationary conditions which actually differ from the optimal processing parameters required for individual conjunctive layers. Usually, the minimum substrate temperature to grow [GGG/BIG] ${ }^{m}$ stack on GGG crystal was higher than commonly used for PLD-made BIG. At such elevated temperatures one has to take care of volatile $\mathrm{Bi}$ re-evaporation. This was the reason to use overstoichiometric target with the Bi excess. Detailed PLD and rf-magnetron sputtering processing parameters were published in ${ }^{3-6)}$ and ${ }^{8,9}$, respectively.

Hereinafter we designate MOPCs as $750-[\mathrm{BIG} / \mathrm{GGG}]^{m}$. 750 stands for the resonance wavelength $\lambda_{0}=750 \mathrm{~nm}, \mathrm{~m}$ is the number of double $\mathrm{Gd}_{3} \mathrm{Ga}_{5} \mathrm{O}_{12} / \mathrm{Bi}_{3} \mathrm{Fe}_{5} \mathrm{O}_{12}$ layers deposited on top of the single crystal substrate to serve as a bottom optical mirror. Next a single $\mathrm{Bi}_{3} \mathrm{Fe}_{5} \mathrm{O}_{12}$ layer follows as an optical cavity and finally a top mirror was deposited with $m$ double $\mathrm{Bi}_{3} \mathrm{Fe}_{5} \mathrm{O}_{12} / \mathrm{Gd}_{3} \mathrm{Ga}_{5} \mathrm{O}_{12}$ layers (see inset in Fig. 4). All the layers in the mirrors have thicknesses of a quarter wavelength $\lambda_{0} / 4 n_{\mathrm{BIG}(\mathrm{GGG})}$ in the respective materials whereas the BIG in the optical resonant cavity has a thickness $\lambda_{0} / 2 n_{\mathrm{BIG}}$. Here $n_{\mathrm{BIG}(\mathrm{GGG})}$ is the refractive index of $\mathrm{BIG}(\mathrm{GGG})$ at the designed wavelength $\lambda_{0}$. Fine adjustment of BIG and GGG thicknesses was performed through a sequence of trial and error fabrications of test film structures

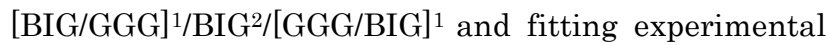
data to transmission and FR spectra calculated by the transfer matrix method. ${ }^{4)}$ 


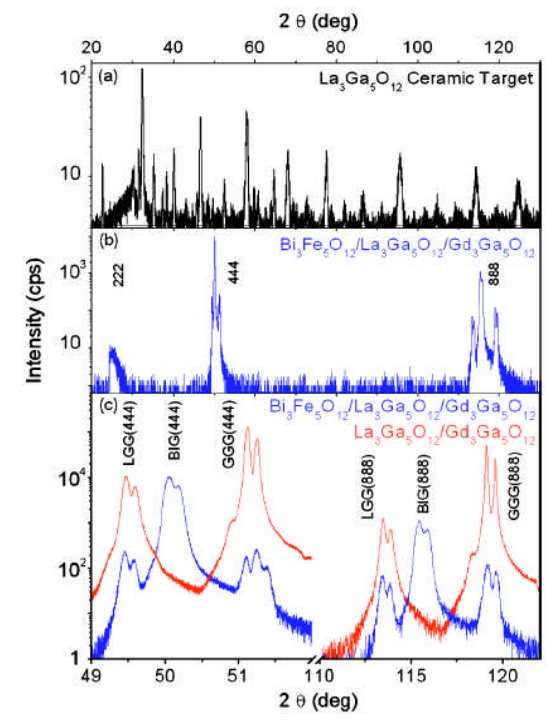

Fig. 1 XRD $\theta-2 \theta$ scans in $\mathrm{Cu}_{\alpha}$ radiation for $\mathrm{La}_{x} \mathrm{Ga}_{y} \mathrm{O}_{z}$ ceramic target (a), $\mathrm{Bi}_{3} \mathrm{Fe}_{5} \mathrm{O}_{12} / \mathrm{La}_{3} \mathrm{Ga}_{5} \mathrm{O}_{12}$ heteroepitaxial film structure (b and $\mathbf{c}$ ), and single-layer $\mathrm{La}_{3} \mathrm{Ga}_{5} \mathrm{O}_{12}$ film (c) grown on the $\mathrm{Gd}_{3} \mathrm{Ga}_{5} \mathrm{O}_{12}(111)$ single crystal.

As an example of achieved epitaxial garnet films quality, Figs. 1 present X-ray diffraction (XRD) patterns of novel $\mathrm{La}_{3} \mathrm{Ga}_{5} \mathrm{O}_{12}$ (LGG) garnet and BIG/LGG bilayer film grown on to the GGG(111) crystal. 5) Bragg reflections of $\mathrm{LaGaO}_{3}$ superimposed with non-reacted $\mathrm{La}_{2} \mathrm{O}_{3}$ and $\mathrm{Ga}_{2} \mathrm{O}_{3}$ oxides were identified for the $\mathrm{La}_{x} \mathrm{Ga}_{y} \mathrm{O}_{12}$ ceramic target in Fig. 1a. Both LGG and BIG/LGG film patterns in Fig. 1b reveal exclusive (111) orientation of garnet films with no other peaks observed. Figure 1c shows the magnified 444 and 888 Bragg reflections which fitted to Nelson-Riley function give cubic lattice parameter $a=12.768 \AA, 12.626 \AA$, and $12.384 \AA$, for LGG, BIG, and GGG, respectively. Rocking curves of 444 Bragg reflections in Fig. 2 show successive narrowing of the full width at half maximum (FWHM $=0.47^{\circ}$ and $0.22^{\circ}$ ) demonstrating the improvement of crystalline properties of BIG film grown on LGG buffered GGG substrate. Inset to Fig. 2 shows

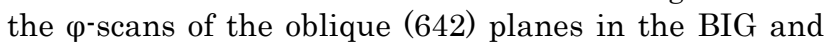
LGG films and GGG(111) substrate. Six (642) reflections from BIG and LGG films appear at the same $\varphi$ angles indicating strong in-plane texture of the heteroepitaxial BIG/LGG structure.

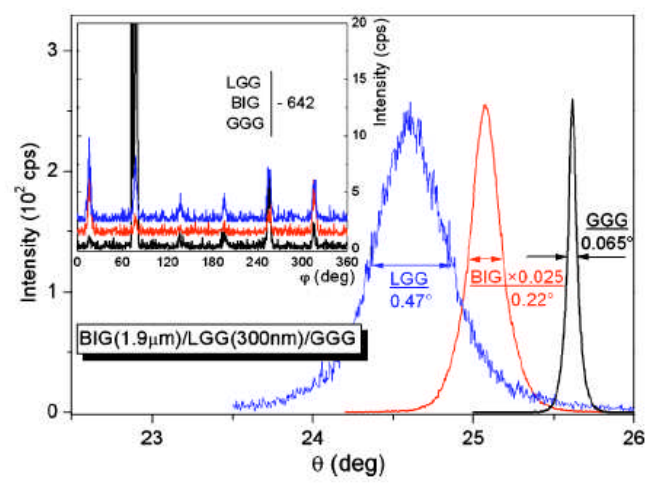

Fig. 2 Rocking curves of the (444) Bragg reflections from the $\mathrm{Bi}_{3} \mathrm{Fe}_{5} \mathrm{O}_{12} / \mathrm{La}_{3} \mathrm{Ga}_{5} \mathrm{O}_{12} / \mathrm{GGG}(111)$ heteroepitaxial structure. Inset: $\varphi^{-}$scans of the oblique (642) planes in the $\mathrm{Bi}_{3} \mathrm{Fe}_{5} \mathrm{O}_{12}, \mathrm{La}_{3} \mathrm{Ga}_{5} \mathrm{O}_{12}$ layers and GGG(111) substrate.

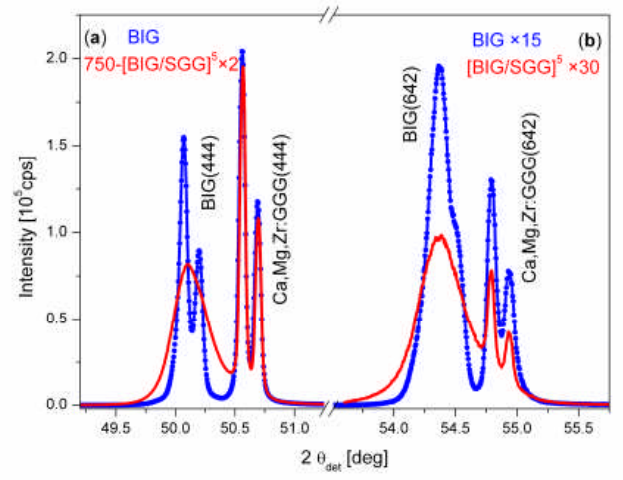

Fig. 3 XRD patterns in $\mathrm{Cu} K_{\alpha}$ radiation of 750-[BIG/GGG $]^{5}$ MOPC (solid line) and the reference $0.7 \mu \mathrm{m}$ thick BIG film (line dotted with $\bullet$ symbols) on to the Ca,Mg,Zr:GGG(111) crystal. Panels a and b: coupled $\theta-2 \theta$ and "uncoupled" $(\theta+\delta \theta)-2 \theta$ scans around (444) and (642) Bragg reflections, respectively.

High precision XRD-examination of $\mathrm{Bi}_{3} \mathrm{Fe}_{5} \mathrm{O}_{12} /$ $\mathrm{Sm}_{3} \mathrm{Ga}_{5} \mathrm{O}_{12}$ MOPCs reveal the character of the strain accumulated in all-garnet multilayers. 9) Figure 3a shows $\theta-2 \theta$ scans in Bragg-Brentano ("coupled") geometry around (444) reflection in the reference 0.7 $\mu \mathrm{m}$ thick BIG film and 750-[BIG/SGG] ${ }^{5}$ MOPC grown on Ca,Mg,Zr:GGG(111) crystal. BIG, Ca,Mg,Zr:GGG substrate and SGG have the lattice parameters in the following order: $12.627 \AA$, $12.498 \AA$ and $12.433 \AA$. Unlike single BIG layer film, MOPC Bragg reflections are inhomogeneously broaden (steep slope at low and gradual at high $2 \theta$ angles) both in "coupled", "un-coupled" $(\theta+\delta \theta)-2 \theta$ scan of the oblique (642) crystallographic plane in Fig. $3 \mathrm{~b}$ and (444) rocking curve in Fig. 4. FWHM of the MOPC rocking curve appeared to be bigger by a factor of four compared to single layer BIG film.

We found that BIG films deposited on to the non-matched (111) garnet substrates experience rhombohedral crystalline distortions. To quantify them we used the off-normal XRD scans of oblique (642) plane in Fig. 3b. In ideal cubic structure the angle $\delta \theta$ between (111) and (642) planes is $22.208^{\circ}$. In our experiments to maximize (642) Bragg reflections $\delta \theta$ was found to be $22.471^{\circ}$ in $750-$ [BIG/GGG $^{5} \mathrm{MOPC}$ and $22.446^{\circ}$ in single BIG layer film. Using standard formulas for interplanar angles we obtained the rhombohedral angles

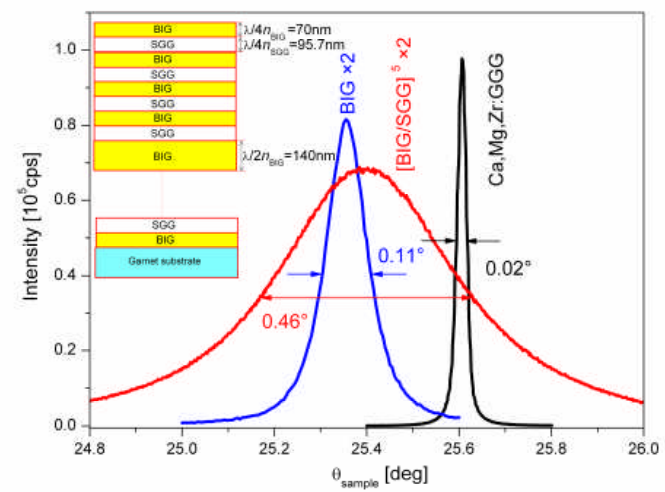

Fig. 4 Rocking curves of (444) Bragg reflections for: 750-[BIG/GGG $]^{5}$ MOPC $\left(\mathrm{FWHM}=0.46^{\circ}\right)$, reference 0.7 $\mu \mathrm{m}$ thick BIG film $\left(0.11^{\circ}\right)$ and $\mathrm{Ca}, \mathrm{Mg}, \mathrm{Zr}$ :GGG substrate $\left(0.02^{\circ}\right)$. Inset shows schematic of magneto-optical photonic crystal 750-[BIG/SGG] ${ }^{4}$. 
$\Psi=88.682^{\circ}$ and $88.826^{\circ}$ that correspond to the rhombohedral distortions of $1.5 \%$ and $1.3 \%$ in 750-[BIG/GGG] 5 MOPC and BIG, respectively.

The rhombohedral distortions give rise to the asymmetric broadening of BIG reflections in MOPC (Fig. 3). Namely, in single layer BIG film mismatch strain relaxes through the net of misfit dislocations embedded in close vicinity (about 10 $\mathrm{nm}$ ) of film/substrate interface. The rest of BIG film is unstrained and gives sharp Bragg reflections with resolved $K_{\alpha 1}$ and $K_{\alpha 2}$ lines (Fig. 3). On the contrary, in multilayered structure regular alternating BIG and SGG layers inhibits the nucleation of misfit dislocations and a long range rhombohedral distortions are preserved through the whole MOPC thickness and cause the gradually shaped high $2 \theta$-shoulders of the Bragg reflections in Fig. 3. Elastic distortions are inhomogeneous within multilayer stack thickness and statistically distributed from non-distorted BIG cubic structure $\left(\Psi=90^{\circ}\right)$ to strongly distorted which enables exact in-plane matching to $\mathrm{Ca}, \mathrm{Mg}, \mathrm{Zr}$ :GGG substrate $\left(\Psi<90^{\circ}\right.$, higher $2 \theta$ angles and shorter $d_{444}$ interplane distance).

\section{Transmission, reflection and Faraday rotation MOPCs' spectra}

Optical dispersion in fabricated garnet films was examined from the transmission and reflection light spectra at normal and oblique incidence. Figures 5 compile transmission $T$, net FR $\Theta_{\mathrm{F}}$ and MO-quality factor $Q=2$ $\left|\Theta_{F}\right| / \ln (1 / T)$ wave length spectra in the reference BIG film and 750-[BIG/SGG ${ }^{m}$ MOPCs with $m=4,5$, and 6 reflectors grown on $\mathrm{Ca}, \mathrm{Mg}, \mathrm{Zr}: \mathrm{GGG}(111)$ substrates. ${ }^{9)} \mathrm{BIG}$ and MOPCs have the same position of the absorption edge at $\lambda_{\mathrm{o}}=542 \mathrm{~nm}(2.29 \mathrm{eV})$. MOPCs' spectra have a stop band

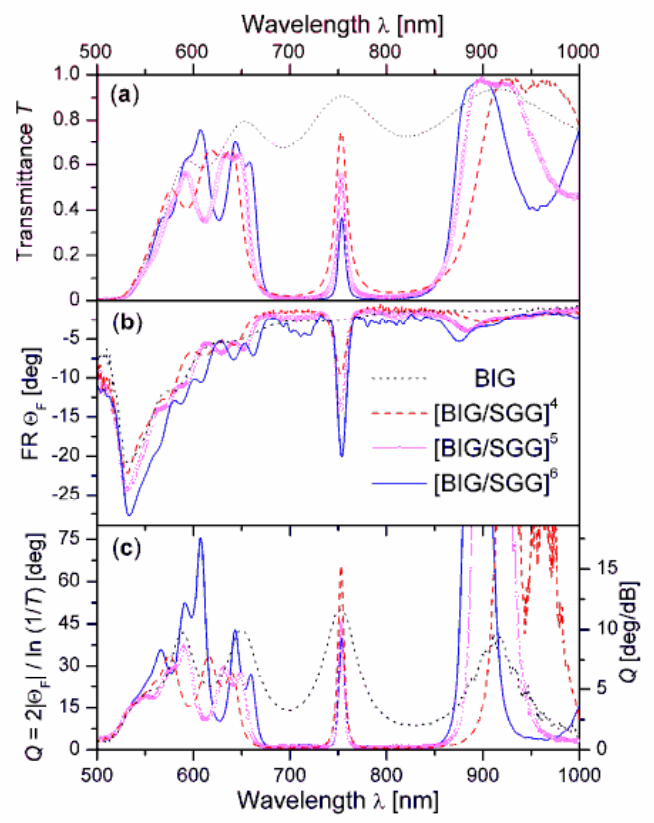

Fig. 5 Transmission $T$, net Faraday rotation $\Theta_{F}[\mathrm{deg}]$ and MO quality factor $Q=2\left|\Theta_{\mathrm{F}}\right| / \ln (1 / T)$ wave length spectra in $750-[\mathrm{BIG} / \mathrm{SGG}]^{4}, 750-[\mathrm{BIG} / \mathrm{SGG}]^{5}, 750-[\mathrm{BIG} / \mathrm{GGG}]^{6}$ MOPCs and reference $0.7 \mu \mathrm{m}$ thick BIG film on $\mathrm{Ca}, \mathrm{Mg}, \mathrm{Zr}: \mathrm{GGG}(111)$ substrates.

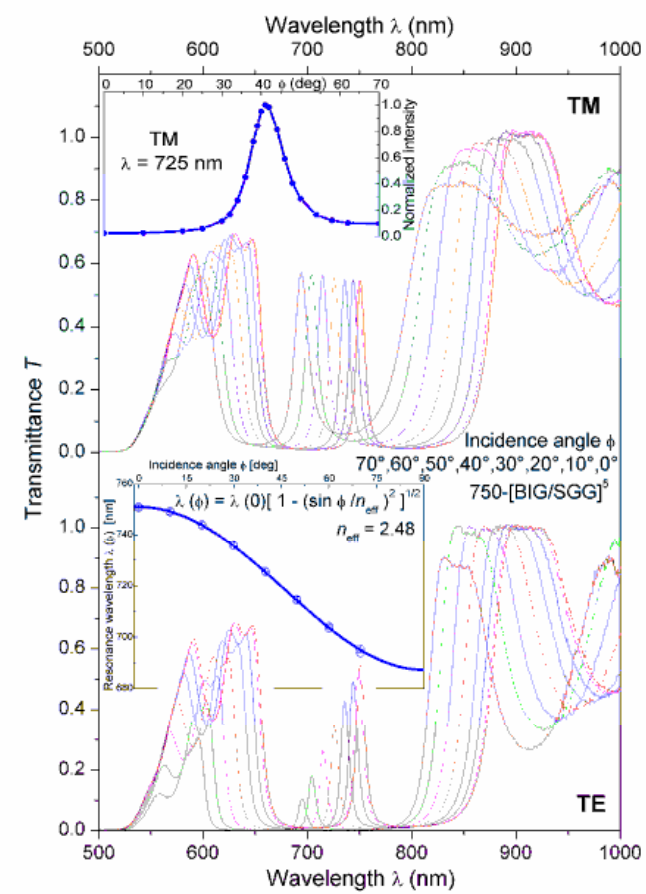

Fig. 6 Transmission spectra for TM- and TEpolarizations in the 750 -[BIG/SGG] $/ \mathrm{Ca}, \mathrm{Mg}, \mathrm{Zr}$ :GGG(111) MOPC at oblique incidence. $\phi$ is the incidence angle between the light beam and the normal to the film surface. Insets: upper - angular dependence of the normalized transmittance in TM-polarization recorded for the wavelength $\lambda=725 \mathrm{~nm}$, lower - angular dependence of the transmission resonance peak position for TE- and TM-polarizations fitted to the Fabry-Perot formula.

structure - the band gap with the transmittance central peak caused by the light localization in the half-wave length BIG cavity. Crystals with bigger number of reflectors $m$ show better light rejection within the band gap: the band edges become steeper, the band gap narrows and earns better squareness, the extinction increases and reaches $19 \mathrm{~dB}$ in $750-[\mathrm{BIG} / \mathrm{SGG}]^{6}$ crystal. Net $\Theta_{\mathrm{F}}[\mathrm{deg}]$ and specific Faraday rotation $\theta_{\mathrm{F}}[\mathrm{deg} / \mu \mathrm{m}]$ also increase with a number of reflectors. $\theta_{\mathrm{F}}$ in [BIG/SGG] ${ }^{6}$ crystal increased by a factor of 6 compared to the FR in one layer BIG film of equivalent thickness (Fig. $5 b)$. Meanwhile, the absorption grows with the number of reflectors faster than the Faraday rotation. This suggests light scattering on the BIG/SGG interfaces contributes to the optical loss. To conclude, MOPCs with higher $m$ provide significant enhancement of FR and narrow bandwidth of the central resonance peak whereas a smaller number of reflectors is still advantageous regarding crystal transparency. Further improvement of MO-properties can be achieved growing MOPCs on better matched substrates. Higher FR and higher transparency was demonstrated in 750 -[BIG/SGG $]^{5} \mathrm{MOPC}$ grown on to the $\mathrm{Gd}_{3} \mathrm{Sc}_{2} \mathrm{Ga}_{3} \mathrm{O}_{12}$ crystal which has bigger lattice parameter $12.570 \AA$ compared to $12.498 \AA$ in $\mathrm{Ca}, \mathrm{Mg}, \mathrm{Zr}: \mathrm{Gd}_{3} \mathrm{Ga}_{5} \mathrm{O}_{12}$ garnet. ${ }^{9)}$

New properties of MOPC spectra appear when light incidents on crystal at oblique angle $\phi$. As expected, the absorption edge around $542 \mathrm{~nm}$ does not change its position. Meanwhile, all the resonant features as edges of the band gap and central peak experience significant (about $56 \mathrm{~nm}$ for 750 -[BIG/SGG]5 crystal) "blue" shift when the incidence angle $\phi$ increases up to $70^{\circ}$ (Figs. 6). The oblique geometry removes the degeneracy of TE- and 
TM-polarizations occurred at normal incidence. For TM-polarization central peak keeps constant height and light rejection within the band gap becomes slightly weaker when $\phi$ angle increases. TE-polarization is characterized by transmittance peak height decrease and much stronger extinction of light within the band gap. Tilting of 750 -[BIG/SGG $]^{5}$ crystal for $70^{\circ}$ from the normal incidence gives $60 \mathrm{~nm}$ band tunability, widens the resonance bandwidth from 8 to $15 \mathrm{~nm}$, and reduces the peak-to-valley extinction ratio from 19 to $14 \mathrm{~dB}$ for TM mode. Upper inset in Fig. 6 shows the angular dependence of the transmittance in 750 -[BIG/SGG] ${ }^{5}$ crystal recorded at $\lambda=725 \mathrm{~nm}$. TE mode conserves the resonance bandwidth of $8 \mathrm{~nm}$, increases the extinction ratio from 19 to $51 \mathrm{~dB}$ whereas light absorption at the transmittance resonance significantly increases from $2.6 \mathrm{~dB}$ at normal incidence to $9.8 \mathrm{~dB}$ at $\phi=70^{\circ}$.

The angular dependence of the resonance wavelength $\lambda(\phi)$ has universal character both for TEand TM-polarizations (lower inset in Fig. 6) and nicely fits to the formula that expresses the condition of constructive light interference in the crystal with effective refractive index $n_{\text {eff }}=2.48$ :

$$
\lambda(\varphi)=\lambda(0)\left[1-\left(\sin \varphi / n_{\text {eff }}\right)^{2}\right] 1 / 2 .
$$

Comparison of transmission and reflection spectra for TM and TE modes is shown in Fig. 7. Light extinction and resonant transparency are always accompanied with enhanced reflectivity within the band gap and opaqueness at the resonance, respectively. Flat transmittance and reflectance of the TM-polarization over the whole range of oblique incidence (resonant omnidirectionality), very strong light rejection and enhanced Faraday rotation demonstrate feasibility to use observed effects for tunable MO-filters in transmission and reflection modes.

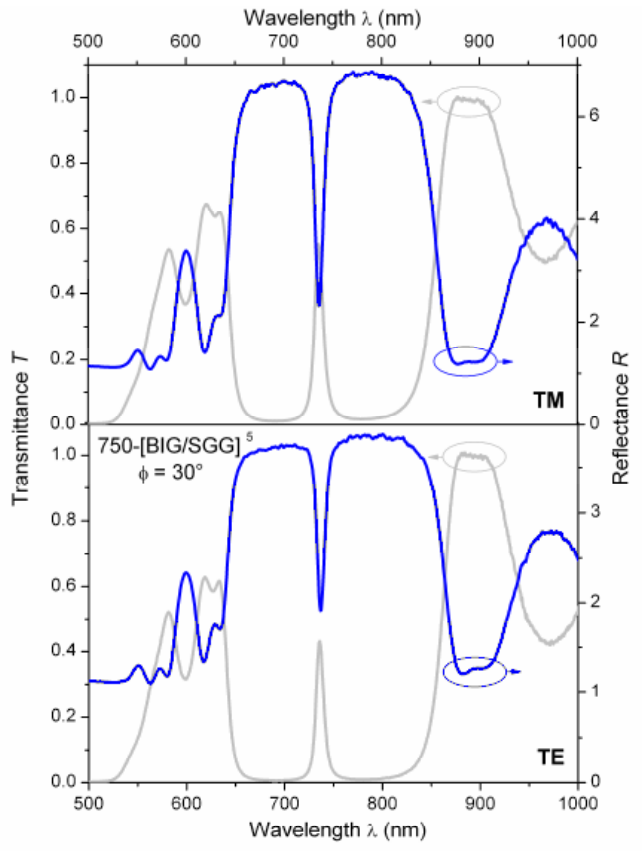

Fig. 7 Transmission and reflection wave length spectra for TM- and TE-polarizations in the 750-[BIG/SGG]5/ Ca,Mg,Zr:GGG(111) MOPC at incidence angle $\phi=30^{\circ}$.

\section{Nanostructured $\mathrm{Bi}_{3} \mathrm{Fe}_{5} \mathrm{O}_{12}: \mathrm{Gd}_{3} \mathrm{Ga}_{5} \mathrm{O}_{12}(n: m)$ superlattices for $\mathrm{MO}$ visualizers and displays ${ }^{7)}$}

To achieve MO remanence combined with enhanced Faraday rotation we sintered by PLD the series of heteroepitaxial all-garnet $\mathrm{Bi}_{3} \mathrm{Fe}_{5} \mathrm{O}_{12}: \mathrm{Gd}_{3} \mathrm{Ga}_{5} \mathrm{O}_{12}(n: m)$ nanostructured MO-superlattices $(n=1,2,3,4,5$ and $m=1$, 2 are the numbers of crystal unit cells). Processing parameters and structure of grown films have been optimized to obtain perpendicular magnetic anisotropy, square hysteresis loop with low coercive and saturation magnetic fields.

To reduce $4 \pi M_{\mathrm{s}}$, hence to achieve perpendicular magnetic anisotropy, we employed BIG and GGG atomic layer deposition. Strong Faraday rotation was preserved in BIG layers whereas the reduction of $4 \pi M_{\mathrm{s}}$ was achieved by dilution of BIG with diamagnetic GGG layers. BIG and GGG garnets have the lattice parameters $a=1.263$ and $1.238 \mathrm{~nm}$, respectively. Due to the lattice mismatch $\delta a / a=-0.020$, BIG on GGG experiences compressive stress $\sigma \sim 0.8 \mathrm{GPa}$. In thick film, the uniform stress accumulates too much elastic energy thus relaxes through the appearance of misfit "dislocation net". The net consists of a system of straight parallel edge dislocations embedded at equal distances $h=a^{2} / \delta a$ from each other in the plane perpendicular to their Burger's vectors $b$. Stress

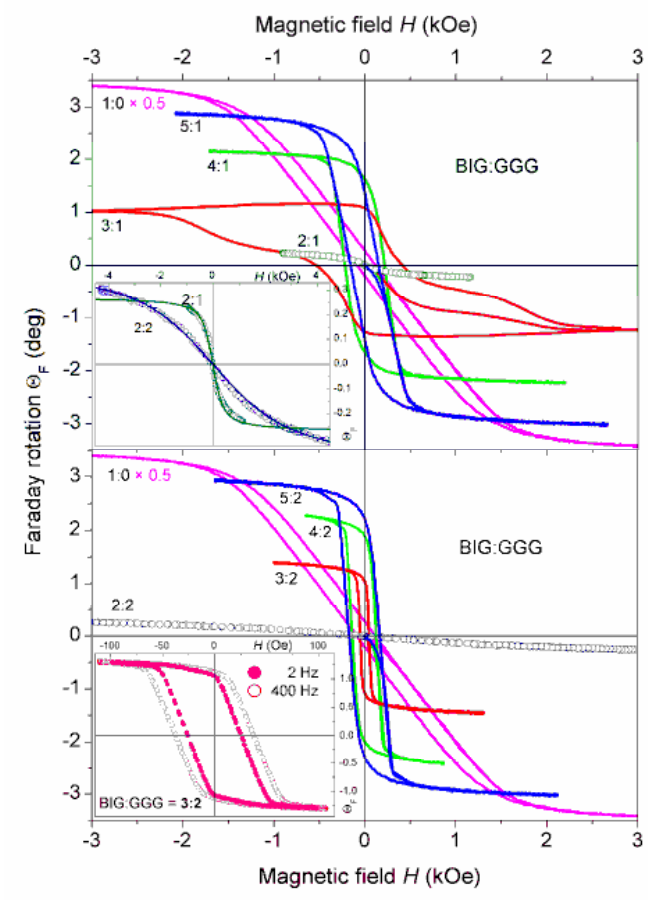

Fig. 8 Hysteresis net FR $\Theta_{F}[\mathrm{deg}]$ vs. perpendicular magnetic field $H$ loops recorded in pulsed magnetometer (raising time $3.9 \mathrm{~ms}$, max magnetic field $20 \mathrm{kOe}$ ) at $\lambda=678 \mathrm{~nm}$. Scaling factor $\times 0.5$ was introduced to plot data for homogeneous $1.5 \mu \mathrm{m}$ thick $\mathrm{Bi}_{3} \mathrm{Fe}_{5} \mathrm{O}_{12}$ film notified as BIG:GGG(1:0). Inset in upper panel presents paramagnetic $\Theta_{\mathrm{F}}-H$ traces for BIG:GGG(2:1) and BIG:GGG(2:2) crystals fitted to the Brillouin function (solid lines). Inset in the lower panel shows $\Theta_{\mathrm{F}}-H$ loops for BIG:GGG(3:2) sample recorded in quasistatic regime $(2 \mathrm{~Hz})$ and at $400 \mathrm{~Hz}$ sweeping rate as all others plots in the figure. 
induced by a single dislocation is a long range and alternates its sign with the azimuthal angle: $\sigma \sim \sin \varphi / r$. Periodicity of the dislocation net causes destructive interference of the strain fields generated by individual dislocations thus the net stress decays exponentially with the distance $z$ from the substrate/film interface inside the film interior reaching its maximum at distance $Z_{\mathrm{cr}}=h / 2 \pi$. 10) In films thinner than $Z_{\mathrm{cr}}$, uniform elastic deformations are energetically favorable and vice versa, in films thicker than $Z_{\mathrm{cr}}$, the appearance of the dislocation net minimizes the elastic energy. For BIG and GGG the estimated critical thickness is $Z_{\mathrm{cr}}<10$ $\mathrm{nm}$. Therefore, the nucleation of misfit dislocations will be completely inhibited in BIG:GGG(n:m) stack containing individual layers much thinner than $Z_{\mathrm{cr}}$. Such superlattice remains strongly compressively stressed. Growth of strained garnet structure is the necessary condition to achieve perpendicular magnetic anisotropy thus the out-of-plane MO remanence. Accumulated elastic stress induces uniaxial magnetic anisotropy $K_{\sigma}$ through the magnetostriction effect. In relaxed thick garnet films $K_{\sigma}$ is localized at the distances of about $Z_{\mathrm{cr}}$ and cannot influence significantly on the net magnetization. To attain global uniaxial anisotropy in cubic garnet hence to change the magnetization from in-plane to perpendicular one we must engineer coherent strain through the total thickness of ferrite film.

This idea was realized in two heteroepitaxial series: BIG:GGG( $(n: 1)$ and BIG:GGG( $n: 2)$ where $n=1,2, \ldots 5$ is a number of $\mathrm{Bi}_{3} \mathrm{Fe}_{5} \mathrm{O}_{12}$ atomic layers alternated by 1 or 2 strata of $\mathrm{Gd}_{3} \mathrm{Ga}_{5} \mathrm{O}_{12}$. The integral thickness of BIG was kept the same for all the films (1200 BIG unit cells, $\sim 1.5 \mu \mathrm{m}$ ) whereas the total BIG/GGG film thickness varied from 3 to $1.8 \mu \mathrm{m}$ for samples BIG:GGG $=2: 2$ and $5: 1$,

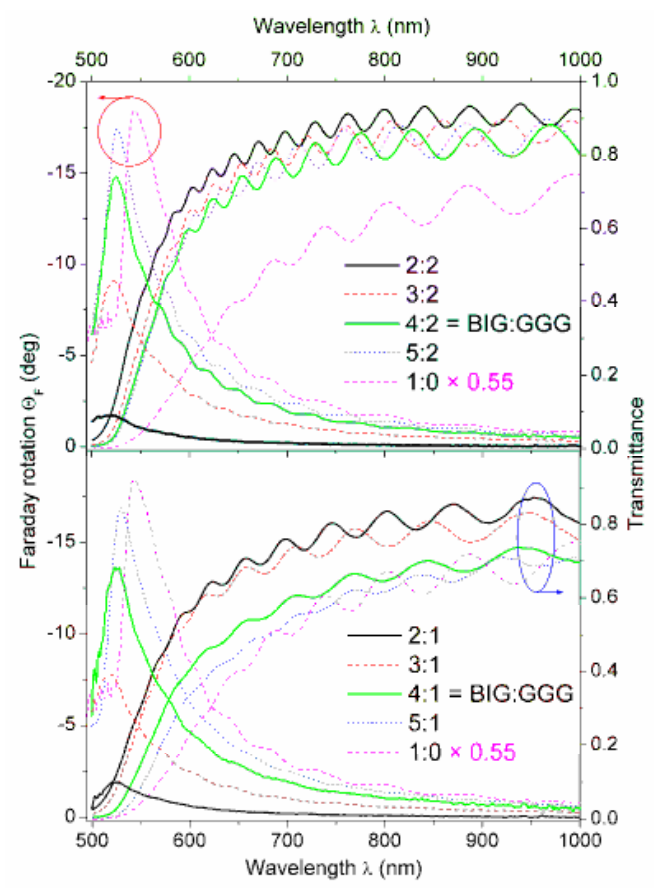

Fig. 9 Transmission and FR spectra for BIG:GGG(n:m) multilayers with the same integral thickness of BIG layers (1200 BIG unit cells, $1.5 \mu \mathrm{m})$. Graphs for the reference $1.5 \mu \mathrm{m}$ thick BIG film (1:0) have been scaled with a factor of 0.55 .

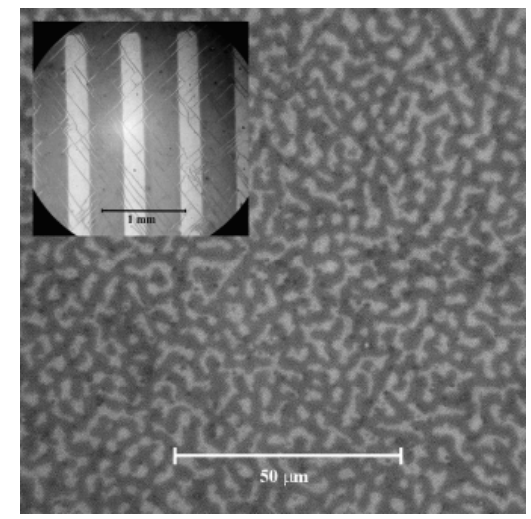

Fig. 10 Magnetic domain structure in $1.9 \mu \mathrm{m}$ thick $\mathrm{Bi}_{3} \mathrm{Fe}_{5} \mathrm{O}_{12}: \mathrm{Gd}_{3} \mathrm{Ga}_{5} \mathrm{O}_{12}(3: 2)$ film (900 BIG unit cells). Inset shows MO-imprint stored in the film brought to the contact with a magnetic bar code in KTH pass card.

correspondingly. Figures 8 show hysteresis FR $\Theta_{\mathrm{F}}-H$ loops recorded by pulsed magnetometer at $\lambda=678 \mathrm{~nm}$ in perpendicular magnetic field while all transmission and FR $\Theta_{F}$ vs. $\lambda$ spectra are collected in Figs. 9.

With decreasing of BIG:GGG ratio FR decreases, films become more transparent and paramagnetic behaviour at room temperature appears in BIG:GGG $=2: 2$ and $2: 1$. Significant enhancement of MO-properties has been achieved in BIG:GGG( $n: 2)$ series mainly due to the enhanced transparency. Flat $Q=2\left|\Theta_{\mathrm{F}}\right| /$ $\ln (1 /$ transmittance $)$ factor as high as $26 \mathrm{deg}$ was obtained for BIG:GGG(2:2) crystal in a wide range from 575 to 775 nm. BIG:GGG(3:2) sample combines high transparency and the lowest saturation and coercive fields: 56 and $25 \mathrm{Oe}$, correspondingly.

Nanostructured BIG/GGG multilayers contain big size $(200-700 \mu \mathrm{m})$ crystallites and magnetic domain structure is easily visualized in polarized light (Fig. 10). Inset shows MO-replica left in the sample brought in a contact with a pass card magnetic barcode. Such MO-imprint can be stored in laboratory for indefinitely long time.

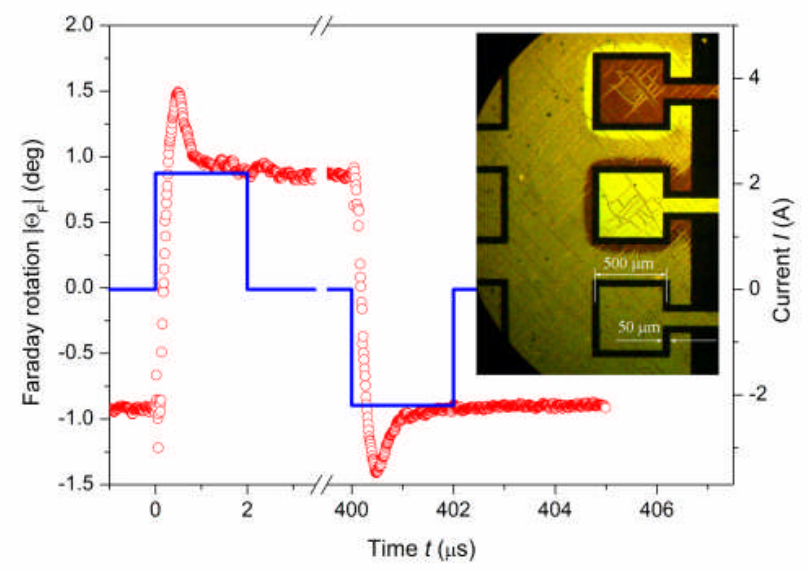

Fig. 11 FR time domain response on $2 \mu$ s pulse of $2.2 \mathrm{~A}$ current fed into the current loop shown in the inset. Before switching, test $1.9 \mu \mathrm{m}$ thick BIG:GGG(3:2) film has been demagnetized. Upper and middle current loops have been exposed to the opposite sign current pulses whereas lower loop (with dimensions shown) has not been powered. 


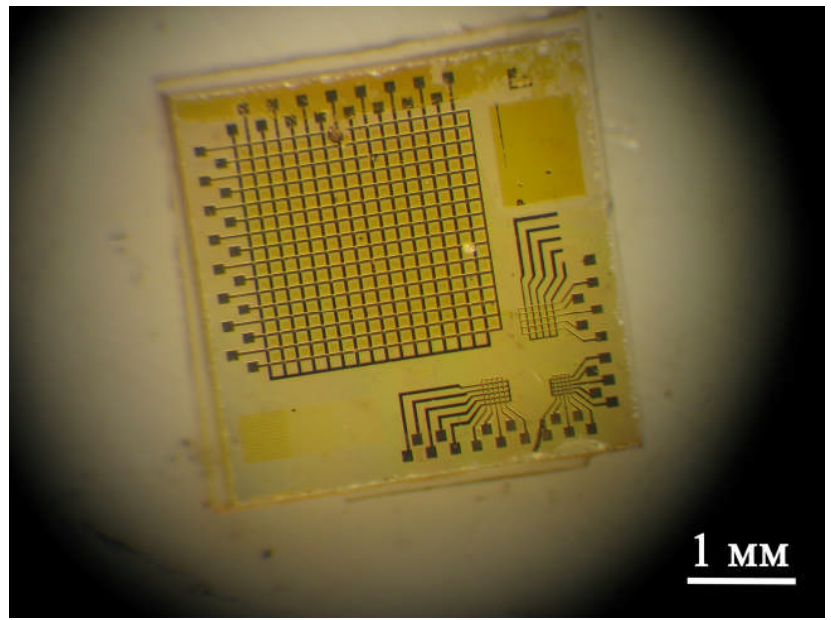

Fig. 12 Test structures of current-driven MO-displays.

Using $1.9 \mu \mathrm{m}$ thick BIG:GGG(3:2) film we also fabricated MO-display containing the array of square $500 \times 500 \mu \mathrm{m}^{2}$ $50 \mu \mathrm{m}$ wide current loops with a typical resistance of 4 $5 \Omega$ (see inset in Fig. 11). The minimal current required to switch MO-pixel inside the loop was about $200 \mathrm{~mA}$. Figure 11 shows time domain FR response on $2 \mu$ s pulse of $2.2 \mathrm{~A}$ current fed into the current loop. Short current pulses produce reproducible high contrast latching type switching of MO-pixels.

Tests structures of current driven MO-displays (spatial modulators) with different pixel size have been fabricated (Fig. 12). Time domain tests are under way to examine switching characteristics of MO-displays.

\section{All-garnet multilayers on glass substrate}

The mechanism of strain accommodation in nanostructured garnet multilayers was utilized to integrate MO-garnets with non crystalline substrates. Crack free $\left[\mathrm{Bi}_{3} \mathrm{Fe}_{5} \mathrm{O}_{12} / \mathrm{Gd}_{3} \mathrm{Sc}_{2.4} \mathrm{Ga}_{2 .} \mathrm{O}_{12}\right]^{m}$ multilayers were grown on to the 7059 Corning glass substrates by rf-magnetron sputtering.

XRD $\theta-2 \theta$ scan of $[\text { GSGG(15nm)/BIG(47.7nm) }]^{13}$ GSGG(15nm) multilayer film contains exclusively Bragg reflections of polycrystalline garnet structure and exactly coincides with XRD pattern of ideal $\mathrm{Gd}_{3}(\mathrm{Sc}, \mathrm{Ga})_{5} \mathrm{O}_{12}$ powder. There was no any splitting of ( $h k l)$ reflections observed even at high angle resolution. Film lattice parameter $a_{0}=12.605 \AA$ has been found using Nelson-Riley refinement procedure. Nanostructured BIG/GSGG multilayers appeared to be coherently strained, lattice parameters in BIG and GSGG layers match each other and lie between the lattice parameters of thick BIG films (12.627 ̊) and GSGG (12.570 ̊).

Optical properties of [GSGG(15nm)/BIG(47.7nm)]13 GSGG(15nm) film are presented in Fig. 13. Total thickness of BIG layers and multilayer film itself was $0.62 \mu \mathrm{m}$ and $0.83 \mu \mathrm{m}$, respectively. At $\lambda=654 \mathrm{~nm}$ specific Faraday rotation $\theta_{\mathrm{F}}=-5.6 \mathrm{deg} / \mu \mathrm{m}$ and transmittance $T=0.67$ demonstrate commercially viable performance of $\mathrm{MO}$ garnets on glass substrate.

\section{Conclusions}

In summary, heteroepitaxial $\mathrm{Bi}_{3} \mathrm{Fe}_{5} \mathrm{O}_{12} / \mathrm{La}_{3} \mathrm{Ga}_{5} \mathrm{O}_{12}$,

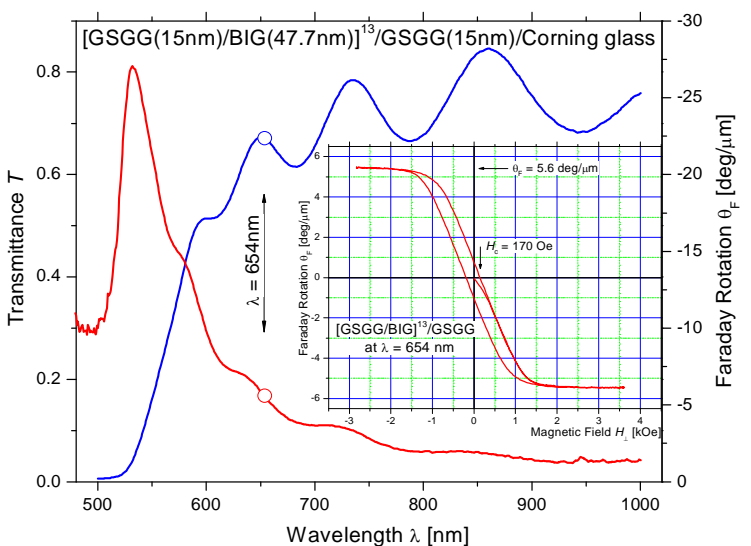

Fig. 13 Transmission and FR spectra of [GSGG(15nm)/ BIG(47.7nm) ${ }^{13}$ GSGG(15nm) multilayer film rfmagnetron sputtered on Corning glass substrate. Symbols $\circ$ mark the wavelength $\lambda=654 \mathrm{~nm}$ used to trace FR vs. magnetic field $H$ hysteresis loop.

$\mathrm{Bi}_{3} \mathrm{Fe}_{5} \mathrm{O}_{12} / \mathrm{Gd}_{3} \mathrm{Ga}_{5} \mathrm{O}_{12}$, and $\mathrm{Bi}_{3} \mathrm{Fe}_{5} \mathrm{O}_{12} / \mathrm{Sm}_{3} \mathrm{Ga}_{5} \mathrm{O}_{12}$ multilayered films grown on available rare earth gallium garnet substrates were shown feasible for various magneto-optical applications. High Faraday rotation and acceptable transmittance in $\mathrm{Bi}_{3} \mathrm{Fe}_{5} \mathrm{O}_{12}$-based magneto-optical photonic crystals were tailored in a wide wavelength range from visible to near infrared. Effect of "blue" shift of transmission spectra in oblique incidence geometry promises photonic crystal as a wide range tunable magneto-optical filter with high light rejection and narrow bandwidth. Atomic garnet layer deposition enables achievement much higher Fe-by-Ga substitution (compared to liquid phase epitaxy) thus coherent within whole film thickness stress-induced uniaxial anisotropy. High performance Faraday rotators combine high MO figure of merit $Q=26 \mathrm{deg}(6 \mathrm{deg} / \mathrm{dB})$ with MO remanence and very low driving magnetic fields. Nanostructured garnets demonstrate potential for MO-imaging, optical signal processing and integration with commercial platforms.

\section{References}

1) M. Levy: IEEE J. Sel. Top. Quantum Electron. 8, 1300 (2002).

2) M. Inoue, R. Fujikawa, A. Baryshev, A. Khanikaev, P.B. Lim, H. Uchida, O. Aktsipetrov, A. Fedyanin, T. Murzina, A. Granovsky: J. Phys. D: Appl. Phys. 39, R151 (2006).

3) N. Adachi, V.P. Denysenkov, S.I. Khartsev, A.M. Grishin, T. Okuda: J. Appl. Phys. 88, 2734 (2000).

4) S. Kahl, A.M. Grishin: Appl. Phys. Lett. 84, 1438 (2004).

5) S.I. Khartsev, A.M. Grishin: Appl. Phys. Lett. 86, 141108 (2005).

6) S.I. Khartsev, A.M. Grishin: Appl. Phys. Lett. 87, 122504 (2005).

7) A.M. Grishin, S.I. Khartsev, S. Bonetti: Appl. Phys. Lett. 88, 242504 (2006).

8) A.M. Grishin, S.I. Khartsev, H. Kawasaki: Appl. Phys. Lett. 90, 191113 (2007).

9) A.M. Grishin, S.I. Khartsev: J. Appl. Phys. 101, 053906 (2007).

10) L.D. Landau and E.M. Lifschitz: Theory of Elasticity (Oxford, Pergamon, 1986).

Received Sept. 12, 2007; Accepted Oct. 29, 2007 